\title{
Pharmacokinetic Concentration Upper Limit Value
}

National Cancer Institute

\section{Source}

National Cancer Institute. Pharmacokinetic Concentration Upper Limit Value. NCI

Thesaurus. Code C124034.

The highest value in a standard range of pharmacokinetic concentration values. 